\title{
A Multistage Control Mechanism for Group-Based Machine-Type Communications in an LTE System
}

\author{
Wen-Chien Hung, ${ }^{1}$ Sun-Jen Huang, ${ }^{1}$ Feng-Ming Yang, ${ }^{2}$ and Chun-Yen Hsu ${ }^{2}$ \\ ${ }^{1}$ Department of Information Management, National Taiwan University of Science and Technology, Taipei, Taiwan \\ ${ }^{2}$ Smart Network System Institute, Institute for Information Industry, Taipei, Taiwan \\ Correspondence should be addressed to Wen-Chien Hung; d10116002@mail.ntust.edu.tw \\ and Feng-Ming Yang; fengmingyang@iii.org.tw
}

Received 18 March 2013; Accepted 17 July 2013

Academic Editor: Anyi Chen

Copyright (C) 2013 Wen-Chien Hung et al. This is an open access article distributed under the Creative Commons Attribution License, which permits unrestricted use, distribution, and reproduction in any medium, provided the original work is properly cited.

\begin{abstract}
When machine-type communication (MTC) devices perform the long-term evolution (LTE) attach procedure without bit rate limitations, they may produce congestion in the core network. To prevent this congestion, the LTE standard suggests using groupbased policing to regulate the maximum bit rate of all traffic generated by a group of MTC devices. However, previous studies on the access point name-aggregate maximum bit rate based on group-based policing are relatively limited. This study proposes a multistage control (MSC) mechanism to process the operations of maximum bit rate allocation based on resource-use information. For performance evaluation, this study uses a Markov chain with $M / G / k / k$ to analyze MTC application in a 3 GPP network. Traffic flow simulations in an LTE system indicate that the MSC mechanism is an effective bandwidth allocation method in an LTE system with MTC devices. Experimental results show that the MSC mechanism achieves a throughput 22.5\% higher than that of the LTE standard model using the group-based policing, and it achieves a lower delay time and greater long-term fairness as well.
\end{abstract}

\section{Introduction}

Machine-type communication (MTC) applications are gradually becoming available for a wide range of potential applications because of the tremendous interest in mobile network operators. This emerging technology is used in machine-tomachine (M2M) communication to provide wireless broadband communications for various types of applications $[1,2]$. This dynamic network provides MTC devices with serving network capabilities for various applications, including metering, road security, and consumer electronic devices $[3,4]$. The 3GPP TS 22.368 specification initially defined the service requirement for MTC services and MTC devices [5, 6]. The SA2 committee extended architecture requirements to support the aggregate maximum bit rate (AMBR) in quality-of-service (QoS) parameters, creating the TR 23.888 specification for group service application requirements [7]. This extension created new challenges and opportunities in the resource allocation problem. The efficiency of the dynamic bandwidth allocation mechanism plays a vital role in system performance because MTC devices can cause network congestion when performing the attach procedure without bit rate limitations [8]. Figure 1 shows a typical MTC network architecture. In a long-term evolution (LTE) system, the mobility management entity (MME) regulates all communication between the enhanced node B (eNB) and MTC devices in the radio coverage cell of the eNB. The policy and charging rules function (PCRF) is a software node for determining policy rules in a 3GPP network. To meet QoS requirements, the MME can send an attachment report to the Home Subscriber Server (HSS), and the HSS attempts to inform the MME by group-based policing to compute the QoS parameter value. To allow all MTC devices to send the attach request separately, the aggregate maximum bit rate (AMBR) must regulate the bit rate of all traffic generated through a group of nonguaranteed bit rate bearers. In this situation, users are unable to receive proper bandwidth, which causes the attach reject for the user equipment-aggregate maximum bit rate (UE-AMBR) and therefore degrades the QoS [9]. 


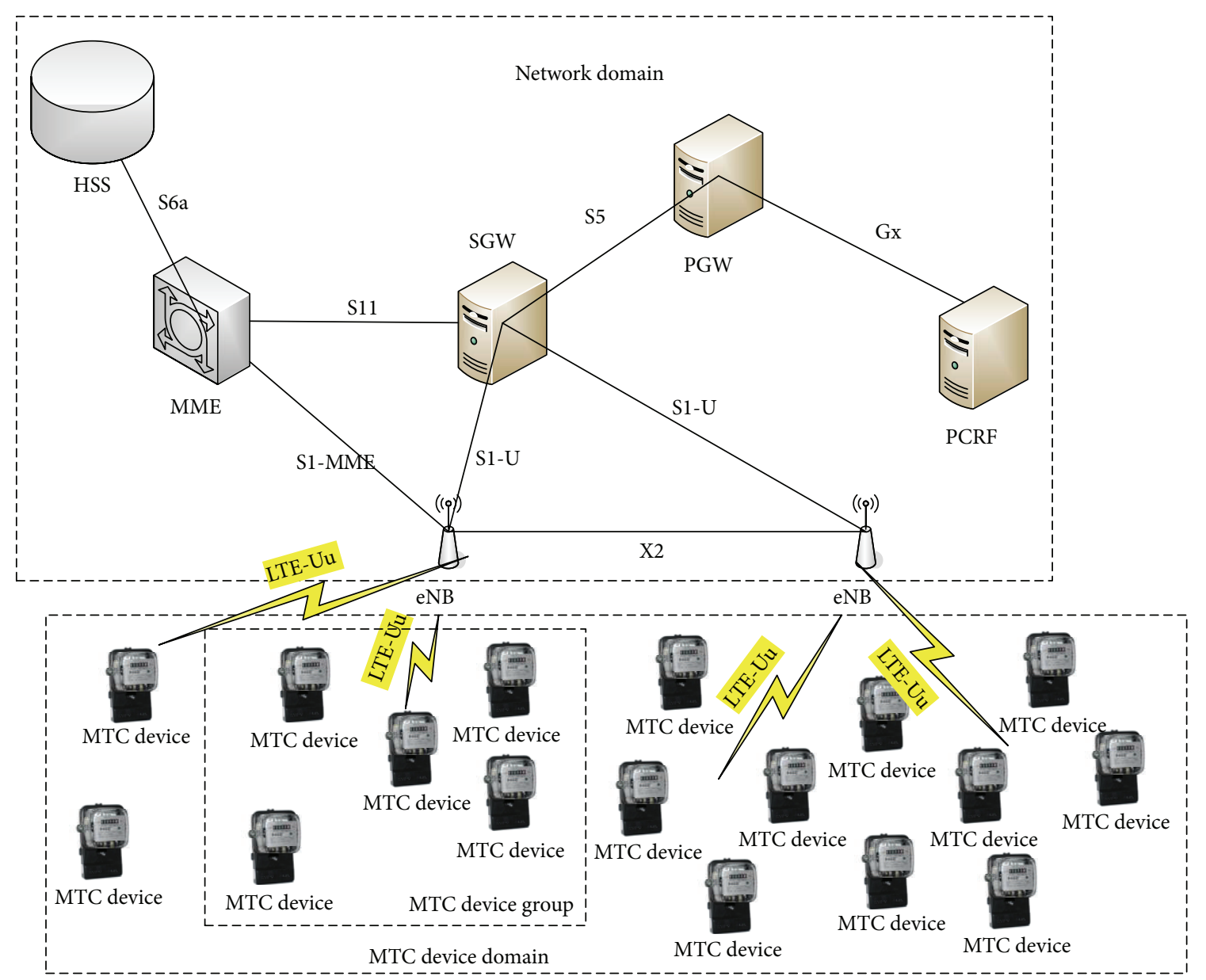

FIgURE 1: MTC architecture in an LTE system.

A crucial bandwidth group-based policing issue in the design of 3GPP networks is the QoS support required for the tremendous global growth in data and traffic of mobile MTC subscribers [10]. A service flow is based on the report information with a particular set of QoS requirement parameters (e.g., packet delay tolerance, acceptable packet loss rates, required minimum bit rates, and AMBR). The LTE system defines several end-to-end QoS requirements and the QoS information for each evolved packet system (EPS) bearer based on the concept of data flows, including the QoS class identifier (QCI), allocation and retention policy (ARP), guaranteed bit rate (GBR), and maximum bit rate (MBR). The QCI is a scalar that indicates a specific priority, maximum delay, and packet error rate. This index also refers to a set of packet-forwarding treatments (e.g., scheduling weights, admission thresholds, queue management thresholds, and link layer protocol configuration). The ARP is involved in prioritization and preemption decisions regarding bearers, and its primary purpose is to decide whether to accept a bearer establishment request when resources are limited. The GBR denotes the minimum bit rate to be provided to a GBR bearer. For a rate-shaping function, the MBR limits the GBR value to discard excess traffic. The MBR and AMBR regulate the bit rate of traffic generated through one GBR bearer and a group of non-GBR bearers, respectively. The LTE system also supports QoS for EPS bearer aggregates at both the UE-AMBR and access point name-aggregate maximum bit rate (APN-AMBR). The UE-AMBR combines the maximum bit rate across all non-GBR bearers of a UE and enforces bandwidth allocation by the eNB for both uplink and downlink. The APN-AMBR aggregates the maximum bit rate across all non-GBR bearers and across all packet data network (PDN) connections of the same APN and enforces bandwidth allocation by the PDN gateway (PGW) for a downlink $[11,12]$.

This study considers MTC devices with an Event-Trigger MTC feature for transmission data only at certain predefined times, which include a grant time interval and a forbidden time interval. The network permits an MTC device to transmit data during the grant time interval and prevents it from transmitting data outside the grant time interval; for example, the grant time interval does not overlap with the forbidden time interval. The network does not permit an MTC device to transmit data during the forbidden time interval for various reasons, such as maintenance of the MTC server. MTC device is to communicate with the MTC server. The communication windows of the devices shall be distributed over the predefined time for a group of MTC devices to avoid network overload. This study focuses on a centralized 
point-to-multipoint (PMP) architecture that the eNB uses to distribute bandwidth resources to multiple MTC devices. This architecture mode provides superior QoS performance to that of the distributed bearer mode. After receiving an attach request from an MTC device, the centralized MME reports an attach report opportunity in time slots to the HSS from all authorized MTC devices currently using the available bandwidth resource.

The remainder of the paper is organized as follows. Section 2 presents a brief literature review of the attach procedure and MTC application over the LTE system. Section 3 provides a description of the system model and the proposed MSC mechanism. Section 4 introduces the simulation environment. Section 5 presents the simulation results of the proposed scheme. Finally, Section 6 offers the conclusion.

\section{Attach Procedures in LTE}

The attach procedure involves one or multiple dedicated bearer establishment procedures to establish a dedicated EPS bearer for that UE. Each bearer is associated with a set of QoS parameters that describes the properties of the transport channel. This leads to a flexible bandwidth allocation algorithm that enables differential treatment for traffic with varying QoS requirements. Network operators allow MTC devices to attach to the network during the predefined period.

2.1. Attach Procedure. In the attach procedure, one UE transmits an attach request message to the eNB. The eNB delivers the initial UE message to the MME to enable session management. The MME provides the serving gateway (SGW) with the UE of the out-of-synchronization (OoS) parameter in the create session request message. In the initial context setup request message, the MME determines the bearer ID and OoS parameter based on the create session information. Session creation management is only defined for the uplink data path, and not for the received downlink data path. The eNB generates the initial context setup response message in response to the initial context setup request message from MME to report the eNB address. The 3GPP standard proposes that the modifying bearer management reports the eNB address to the SGW, enabling the PGW to determine the downlink data path. The UE obtains the downlink data according to the modifying bearer management [13].

The Policy Control Enforcement Function (PCEF) sends an Internet Protocol Connectivity Access Network (IP-CAN) session modification message if the IP-CAN QoS exceeds the authorized QoS provided by the PCRF. The dedicated bearer establishment contains the create bearer and bearer setup messages without data payload. The PGW sends a create bearer request message (while maintaining the OoS parameter) to the MME by triggering a dedicated bearer establishment. When the MME is ready to switch the bearer to dedicated bearer activation mode, it sends a bearer setup request message to the eNB. After the eNB receives the bearer setup request message, it replies with a bearer setup response message. To report the bearer setup response message, the eNB sends its address to the MME without any
QoS parameters. The reported effective bearer setup feedback is consistent with the RRC connection reconfiguration. The PCEF can then be obtained by the optimal QoS report to meet the specified target error rate before the dedicated bearer establishment.

Figure 2 shows a sequence diagram of the attach procedure. For the enabled service, a UE must register with the network by network attachment. The eNB derives the MME from the initial UE message carrying the attach request and the PDN connectivity. The MME sends a create session request (PGW address, APN, default EPS bearer QoS, APNAMBR, etc.) message to the selected SGW. The SGW sends a create session request message to the PGW using the PGW address received in the previous message. The PGW considers the received message and returns a create session response (PDN type, PDN address, EPS bearer ID, EPS bearer QoS, APN-AMBR, etc.) message to the SGW. The eNB sends the $\mathrm{RRC}$ connection reconfiguration message including the EPS bearer ID and attach accept message to the UE, and the $\mathrm{UE}$ returns the RRC connection reconfiguration complete message to the eNB. The MME sends a modify bearer request (EPS bearer ID, eNB address, etc.) message to the SGW, and the SGW acknowledges by sending a modify bearer response (EPS bearer ID) message to the MME.

2.2. Event-Trigger MTC Applications. Although some existing MTC applications use short-range radio technologies, MTC solutions based on mobile access technologies are easier to install. Mobile access-based MTC solutions are also better suited to supporting MTC applications, which require reliable delivery of data to distant MTC devices [14]. Figure 3 shows a MTC application that consists of a modified time and reported device ID at the S6a interface. The MTC device performs initial attach and authentication procedures. When the MME is unaware of the context information of the MTC device, it sends a time period report request, which includes a device ID, to the HSS. After receiving the time period report request from the MME, the HSS determines the period of the MTC device. The HSS returns a time period report, which includes the device ID and time period information of the MTC device, to the MME. To avoid network signaling overhead, the MME calculates the modified grant time interval and determines whether the attach request is from the MTC device. When the attach request is received outside the grant time interval, the MME sends an attach reject message to the MTC device [7]. After an attach request message is accepted, the MME sends a time period report request to the HSS. The time period report request includes a device ID and QoS parameters corresponding to the MTC device making the initial attach request. When the received attach report corresponds to an initial attach report, the HSS determines an APN-AMBR by dividing the group-APN-AMBR assigned to the MTC group by using the group-based policing method. After applying this group-based policing method, the HSS sends an APNAMBR report, which includes the determined APN-AMBR, to the MME. Finally, the MTC device establishes a PDN connection [9]. 


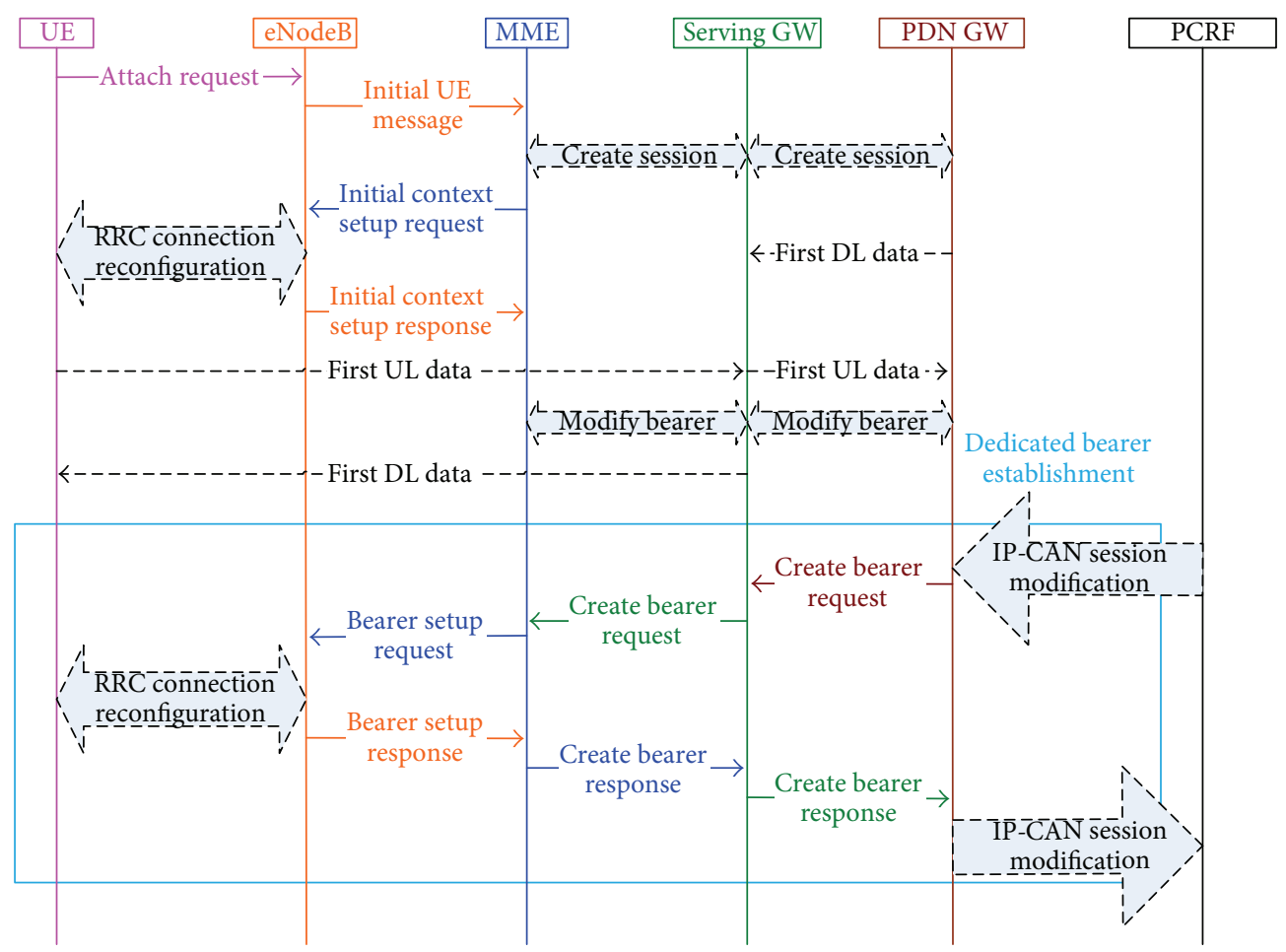

FIGURE 2: Sequence diagram of LTE attach procedure.

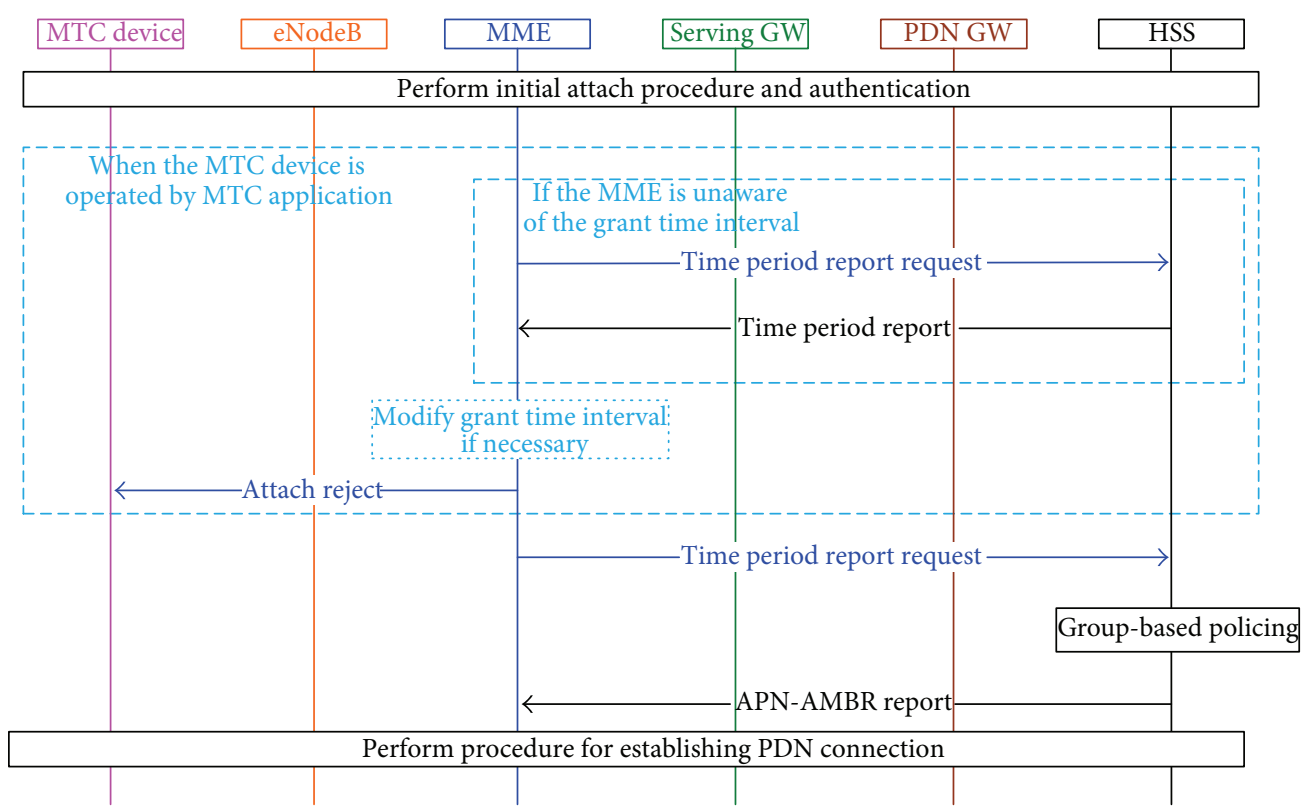

FIGURE 3: Sequence diagram of MTC application in an LTE system.

\section{Proposed Model and Mechanism}

This section presents an analysis of the blocking probabilities of a MTC application that distinguishes multiple classes of equal mean grant time interval. Based on the group-based policing, the HSS uses extended APN-AMBR report fields in 3GPP networks. As the APN-AMBR parameter travels through an MME, UE-AMBR losses occurrence for various APN-AMBR types because of inaccessible bandwidth after the group-based policing. Let $T$ be the grant time interval. Let $G$ be the group-APN-AMBR to group MTC devices when they are served, which is the parameter to fulfill 


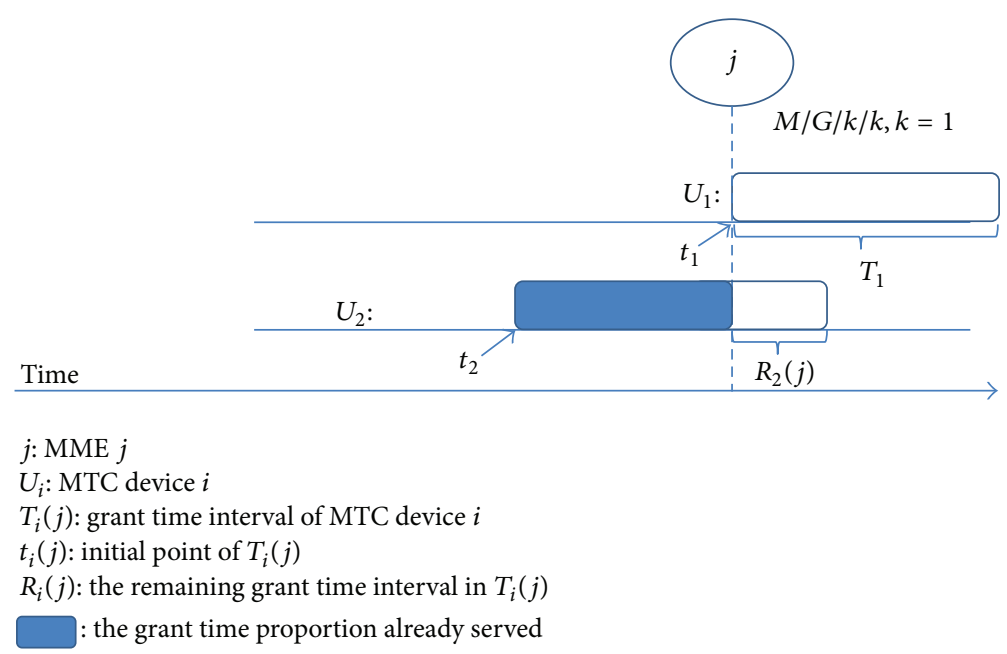

FIGURE 4: Corresponding queuing model for MTC application.

the satisfaction of the bandwidth requirement of application. The terms $T$ and $G$ are the two operational parameters of the proposed framework.

\subsection{Queuing Model for MTC Application. The MTC Appli-} cation being considered is Event-Trigger MTC. Assume the requests arrive at the MME following a Poisson process. Let $N$ be the number of all MTC devices in the network. If the MME can modify the service time, the system behaves as an $M / G / k / k$ system, and the blocking probability can be obtained using the Erlang B formula [15]. However, when the system includes more than one priority class, the application of $M / G / k / k$ becomes more complex. Assume that the system contains two MTC devices. Figure 4 shows the case when MTC Device $2\left(U_{2}\right)$ arrives at the MME before MTC Device $1\left(U_{1}\right)$. However, $U_{2}$ is stopped because the priority ensures the grant time interval of $U_{1}$ by modifying the grant time interval of $U_{2}$. The remaining portion of the overlapping grant time interval at MME $j$ of MTC device $i$ is denoted by $R_{i}(j)$, and this represents the residual service time. If the basic MTC device and all QoS MTC devices are constant, the degree of isolation between two arbitrary classes depends only on their effective overlapping grant time interval. This is because a basic MTC device can be interpreted as a constant shift in time of the reservation process, and thus, neither arrival nor reservation events are reordered in the grant time interval. This result has also been proven by simulation for various arrival and service time distributions. Hence, assume that $R_{i}(j)=0$ without loss of generality and consider the overlapping grant time interval between MTC Device 1 and MTC Device 2 as $R_{2}(j)=T_{2}-\left(t_{2}-t_{1}\right)>0$. In this case, $T_{1}(j)$ has preemptive priority over $R_{2}(j)$. The blocking probability of $U_{1}$ is simply obtained using the Erlang B formula. This study uses the Erlang B formula to evaluate the blocking probability of the all the $T_{i}(j)$ traffic of MTC devices.

Consider a time period report request arriving at a certain time instant in the HSS and requesting to reserve a grant time interval $q$ time slots after its arrival time. Without loss of generality, consider the arrival time of the time period report request to be slot 0 , and the start of the MTC device requested duration to be slot $q$. To calculate the blocking probability of this MTC device request, consider the traffic load at slot $q$, as seen at the time of the request. Any MTC device requested duration generated in the future (from time slots after slot 0 ) for slot $q$ will not affect the probability of accepting/blocking the MTC device request. The number of grant time intervals with a start time within slot $q$, as seen at the instant in which the time period report request arrives, is Poisson-distributed with mean

$$
\lambda^{(q)}= \begin{cases}\lambda, & \text { for } q \leq 0, \\ \lambda\left\{1-\sum_{a=0}^{q-1} f(a)\right\}, & \text { for } q>0 .\end{cases}
$$

The number of MTC device requests for time slot $q$ from all previous slots, including slot $q$, is $\lambda$. Given a time period report request arriving in time slot 0 , the probability that this MTC device requested duration requests that time slot $q$ is $f(q)$. Therefore, the number of MTC device requests from slot 0 for slot $\mathrm{n}$ is $\lambda f(q)$. From a time period report request viewpoint, the number of grant time interval arrivals in slot $\mathrm{n}$ is the sum of MTC device requests from all time slots before slot 0 , in addition to the MTC device requests from slot 0 . Thus, the number of arrivals in slot $q$, as seen by the time period report request, is the sum of all possible MTC device requests $(\lambda)$ minus any future MTC device requests to be made in time slots 1 to $q$ for slot $q$.

Based on this assumption, define $\mu_{i}=1 / E\left[T_{i}\right]$, where $E\left[T_{i}\right]$ is the expected value of the MTC device $i\left(U_{i}\right)$ grant time interval at the same MME. To determine the effective service time of a grant time interval under the MTC application, refer to Figure 4. For a predefined time, a bandwidth is reserved for a length of time that is equal to the sum of two time intervals. The duration of the first interval is equal to that of the grant time interval and is distributed according to $T_{i}(j)$ with a mean $1 / \mu$. The conventional Markov model was implemented to analyze the MTC requests queue model. The Markov model 


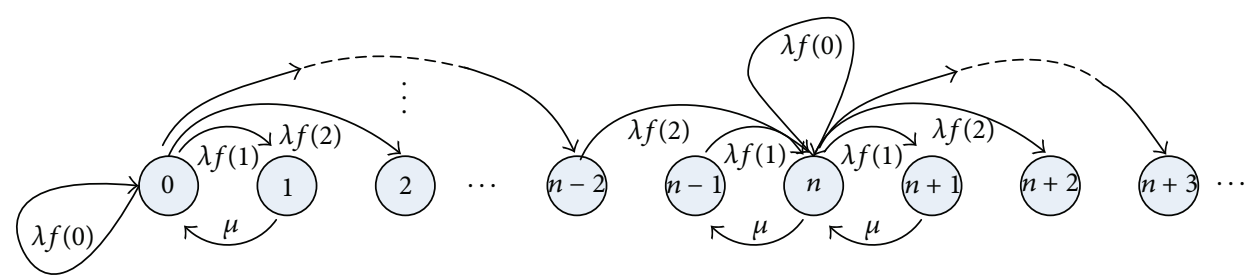

FIGURE 5: Markov model for the MTC requests queue.

was constructed for the MTC requests queue. The state $n$ represents the number of MTC requests within the frame duration. The process of the MTC requests queue assumes that time slot $q$ is working. The Markov process is illustrated in Figure 5, based on the assumptions in our study. The state $n$ represents the number of requests in the MTC system.

The duration of the second interval is equal to that of the forbidden time interval and is distributed according to $R_{i}(j)$ with a mean $\bar{R}$. Based on these observations, an output port of an MME node using a MTC application behaves as an $M / G / k / k$ loss system. The traffic intensity $\rho^{(\mathrm{TC})}$ of the queue is

$$
\rho^{(\mathrm{TC})}=\lambda^{(q)}\left(\frac{1}{\mu}+\bar{R}\right) .
$$

This study first presents modeling the MME using an $M / M / k / k$ queue with preemptive priorities, where the arrival rate of $t_{i}$ is $\lambda_{i}$ and the service rate is $\mu_{i}$. Let $k$ be the number of classes in the MTC application. Denote $\rho_{i}=$ $\left(\lambda_{i} / \mu_{i}\right)$ as the traffic load of $t_{i}$. If $t_{1}$ has an absolute priority (i.e., all other MTC devices have signaling overhead), the blocking probability of one class can be obtained using the Erlang B formula in the $M / M / k / k$ queue, expressed as

$$
P\left(\rho_{1}, k\right)=\frac{\left(\rho_{1}^{k} / k !\right)}{\left(\sum_{m=0}^{k} \rho_{1}^{m} / m !\right)} .
$$

Similar to (3), the blocking probability of the superposition of the two classes with total traffic load $\rho_{1}+\rho_{2}$ can be calculated as follows:

$$
P\left(\rho_{1,2}, k\right)=\frac{\left(\rho_{1,2}^{k} / k !\right)}{\left(\sum_{m=0}^{k} \rho_{1,2}^{m} / m !\right)},
$$

where $\rho_{1,2}=\rho_{1}+\rho_{2}$. In the multiclass case for the $M / G /$ $k / k$ queue, the blocking probabilities for service classes with different QoS values can be obtained by heuristically generalizing (3) and (4) to an arbitrary number of $k$ classes. A conservation law can be formulated for every set of classes $E_{x}=\{0, \ldots, x\}$ with $0<x \leq k-1$ :

$$
\left(\sum_{y=0}^{x} \lambda_{i}\right) P\left(\rho_{1,2, \ldots, E_{x}}, k\right)=\sum_{y=0}^{x} \lambda_{y} \cdot P\left(\rho_{1,2, \ldots, y}, k\right),
$$

where $P\left(\rho_{1,2, \ldots, E_{x}}, k\right)$ is the total blocking probability of all classes in $E_{x}$. It describes the probability that a low-priority MTC device that started the grant time interval prior to the grant time interval of other MTC devices has not finished its grant time interval. In general, the blocking probability of a MTC application can be calculated based on (3)-(5) as follows:

$$
\begin{aligned}
P\left(\rho_{1,2, \ldots, E_{x}}, k\right) & \\
= & \left(\frac{1}{\lambda_{E_{x}}}\right)\left(\sum_{y=0}^{E_{x}} \lambda_{y}\right) \\
& \times\left[P\left(1,2, \ldots, E_{x}\right)-p_{1,2, \ldots, E_{x}-1} P\left(1,2, \ldots, E_{x}-1\right)\right],
\end{aligned}
$$

where $p_{1,2, \ldots, E_{x}-1}=\left(\sum_{y=1}^{E_{x}-1} \lambda_{y}\right) /\left(\sum_{y=1}^{E_{x}} \lambda_{y}\right)$ and $P(1,2, \ldots$, $\left.E_{x}\right)=\left(\left(\sum_{y=1}^{E_{x}} \rho_{y}\right)^{k} / k !\right) /\left(\sum_{m=0}^{k}\left(\sum_{y=1}^{E_{x}} \rho_{y}\right)^{m} / m !\right)$.

3.2. Multistage Control Mechanism. The attach report message is selected from each MTC device after reaching the HSS because the MTC application identifies acceptable attach MTC devices. The goal of the MSC mechanism is to improve throughput requirement with a QoS guarantee. Figure 6 shows a flowchart of the APN-AMBR allocation process. The MME receives an attach request and determines whether a MTC application operates the attached MTC device. If a MTC application operates the MTC device, the MME also determines whether to modify the grant time interval. The MME sends an attach report to the HSS, and the HSS collects all MTC devices requesting attachment. The HSS determines whether a new attach report has been received from the MME. When the HSS knows the already attached MTC device, it can process the MSC mechanism without waiting for a duration. The HSS sends the APN-AMBR report information after the MSC mechanism. The current AMBR definitions enable system operators to differentiate the service level provided for each of these services. In the proposed architecture, rate policing prevents the network from becoming overloaded and ensures that the services send data in accordance with the specified maximum bit rates. Because MTC devices are encouraged to adapt their APNAMBR to starvation, resource starvation is confined to those who ignore starvation. The uplink and downlink scheduling functions implemented by the LTE system are largely responsible for fulfilling the QoS characteristics associated with the different bearers.

These APN-AMBRs are APN-level quantities and are therefore known at the HSS. These APN-AMBRs propagate through UE attach procedures down to the MME to enforce 


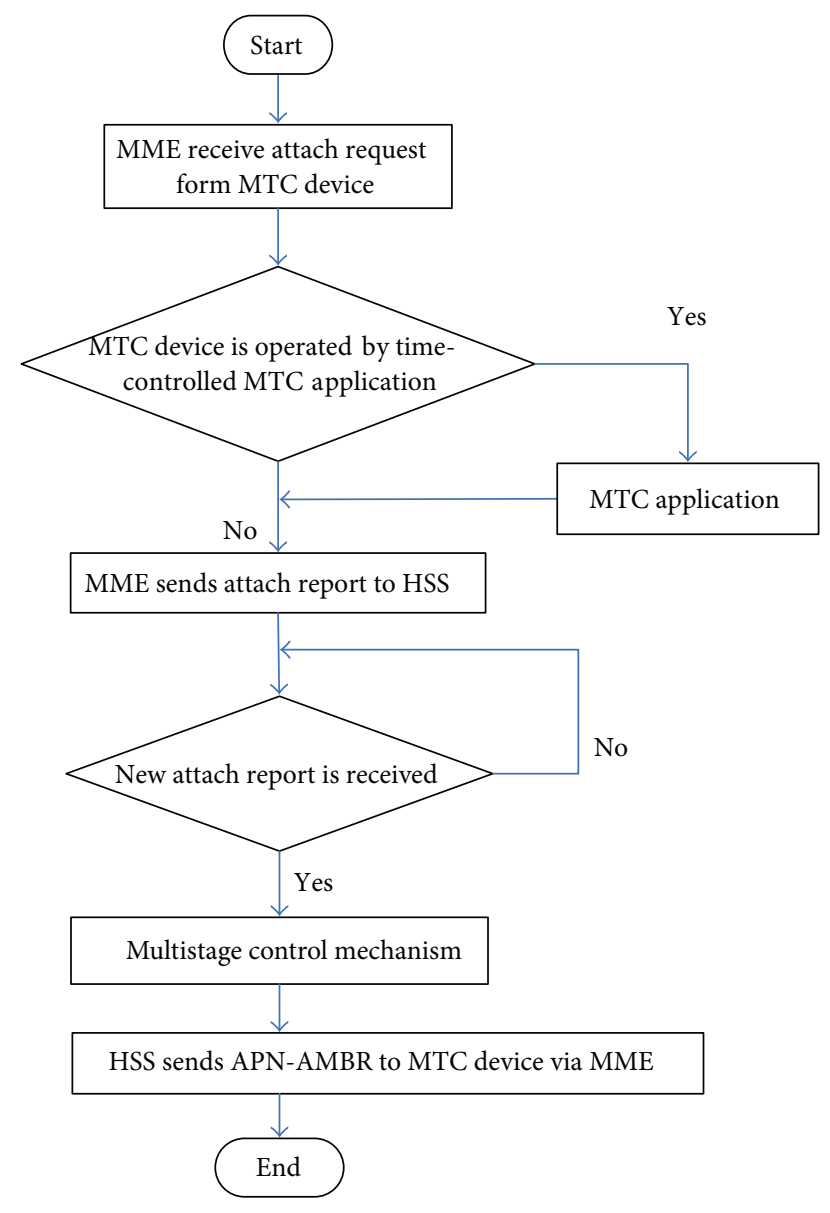

FIGURE 6: Flowchart of the APN-AMBR allocation process.

the data rate from the specific APN through rate policing. Each MTC device may have various packets, and the stream types of these packets may have various UE-AMBRs. This mechanism also uses the following parameters:

(i) $\mathrm{N}$ : the number of MTC devices that must be buffered within an MME in the LTE system;

(ii) Q: the number of MMEs in a MME pool area;

(iii) $S$ : the number of stages in the MSC mechanism;

(iv) $G_{i, j}$ : the group APN-AMBR size of the $i$ th MTC device at the $j$ th MME;

(v) $R_{i, j}$ : the UE-AMBR size of the $i$ th MTC device at the $j$ th MME;

(vi) $D_{i}$ : the default APN-AMBR size of the $i$ th MTC device;

(vii) $M_{i}$ : the minimum APN-AMBR size of the $i$ th MTC device;

(viii) $A_{i}$ : the allocation APN-AMBR size of the $i$ th MTC device.

Consider the MSC mechanism in which resource use can be calculated. Let $C_{i, j}$ be the resource use of the $i$ th MTC device at the $j$ th MME, where $C_{i, j}=\left(R_{i, j} / G_{i, j}\right)$. The challenge of this utility maximization problem is to determine the APNAMBR of each subscribed MTC device to be served and the amount of resources to be allocated to each APN-AMBR of all subscribed MTC devices. When resources are limited, the HSS must determine the number of APN-AMBRs for each MTC device to receive resources, in order to maximize the total utility of the system. We could find an allocation $\Gamma=$ $\left\{A_{1}, A_{2}, \ldots, A_{N}\right\}$, where $A_{i}=\left\{a_{i, j}: a_{i, j} \geq 0, j=1,2, \ldots, Q\right\}$ denotes the set of allocated resource to each APN-AMBR of the $i$ th MTC device. Formally, the objective of this problem is to find a $\Gamma$ to:

$$
\begin{array}{ll}
\text { Maximize : } & \sum_{j=1}^{Q} \sum_{i=1}^{N} R_{i, j} C_{i, j} \\
\text { Subject to }: & \sum_{j=1}^{Q} \sum_{i=1}^{N} a_{i, j} \leq A, \\
& 0 \leq C_{i, j} \leq 1, \\
& R_{i, j} \geq 0, \quad \forall i, j .
\end{array}
$$

By solving this equality, the MTC application can easily be obtained for the user. Resource use can be maximized with the optimal values, which can be obtained by $R_{i, j}$ and $C_{i, j}$ parameters. However, the solution of this optimization equation is not explicit because the MME does not know the group APN-AMBR size and the number of MMEs in the MME pool area in this stage. How to evaluate the APNAMBR for each MTC device is important. One way to address this is to refer to resource use, by employing the utilization range to evaluate the APN-AMBR for each MTC device at the HSS. The utilization ranges for various stages exhibit up-bound and low-bound utilization. Let $H_{s^{\prime}}$ and $L_{s^{\prime}}$ be the up-bound and low-bound utilization of the $s^{\prime}$ th stage, respectively. Define up-bound utilization as

$$
H_{s^{\prime}}= \begin{cases}\sum_{i=1}^{s^{\prime}}\left(\frac{1}{e}\right)^{i}, & \text { if } s^{\prime}<S, \\ 1, & \text { if } s^{\prime}=S .\end{cases}
$$

The up-bound utilization and low-bound utilization can be determined by the HSS. Hence, low-bound utilization can be represented as

$$
L_{s^{\prime}}= \begin{cases}0, & \text { if } s^{\prime}=1, \\ \sum_{i=2}^{s^{\prime}}\left(\frac{1}{e}\right)^{i-1}, & \text { if } 1<s^{\prime}=S .\end{cases}
$$

This method focuses on improving resource utilization. The MSC mechanism helps the MME allocate the APNAMBR for each MTC device to maximize the resource use and satisfy the target UE-AMBR. The granted bit rate of the APN-AMBR is a critical parameter in the MSC mechanism. First, the MME assigns the initial resource use levels, $H_{s^{\prime}}$ and $L_{s^{\prime}}$, based on the required resource use of up bound and low bound, respectively. Therefore, it is necessary to check all utilization ranges $\left(H_{s^{\prime}}, L_{s^{\prime}}\right)$ to find the utilization ranges 


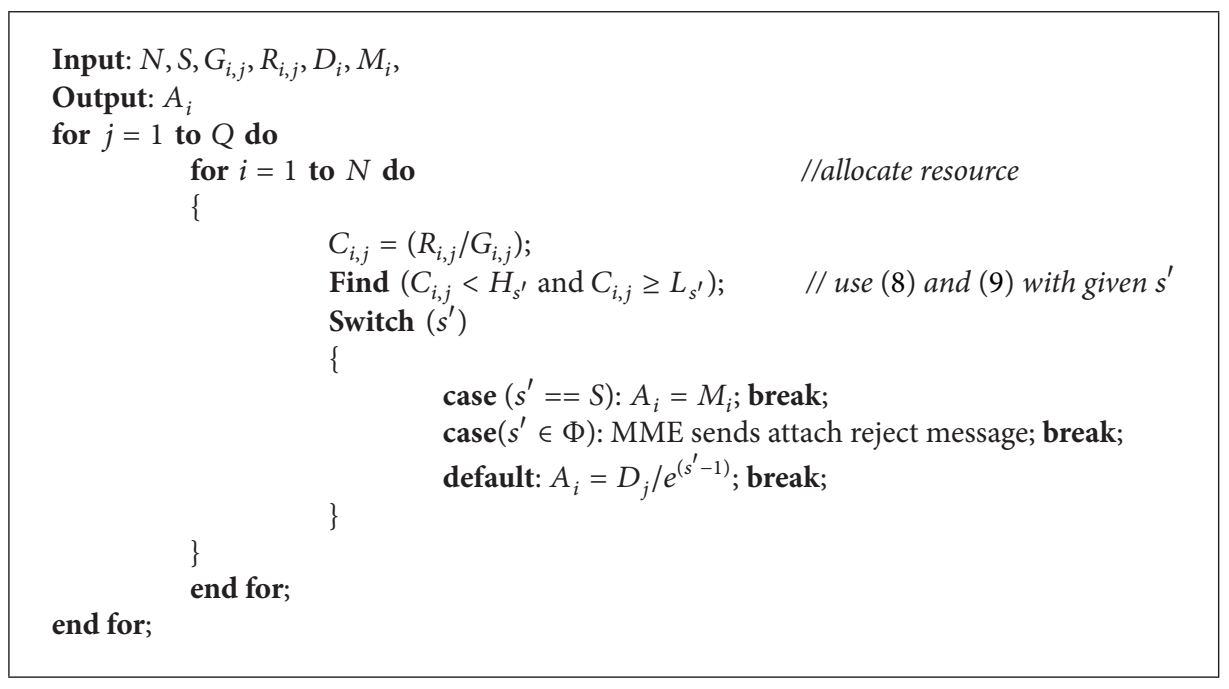

Algorithm 1: Multistage control mechanism.

that maximize resource use as candidate solutions. Among these candidates, the candidate with the maximum resource utilization is the solution for the MSC decision. If multiple combinations produce the same maximum resource use, select the one with the maximum number of MTC devicerejected attachments. Algorithm 1 describes the assignment of the APN-AMBR by using the MSC mechanism in an MME pool area.

To improve fairness, the MTC devices should be allocated their APN-AMBR from the group APN-AMBR in order. If the UE-AMBR of the signaling overhead type is incomplete, signaling nonoverhead UE-AMBR will occupy the resource of the group APN-AMBR in the MTC applications. The MTC device allocation process ensures that FCFS scheduling is based on fairness if the stream types have the same priorities. The frame allocation process guarantees higher throughput and achieves fairness between several MTC device stream types. For this reason, MTC devices with a better resource condition enjoy better perceived quality between several stream types. Jain's fairness index is a conventional method of assessing the quality of the traffic type [16]. The term $f$ represents the total number of QCIs for the duration of the MTC application. The value of Jain's fairness index is generally between $1 / f$ and 1 . When the LTE system indicates an increase in Jain's fairness index value, the system has higher fairness for all CQIs. This model can be written as follows:

$$
\mathrm{FI}=\frac{1}{f} \times \frac{\left(\sum_{n=1}^{f} X_{n}\right)^{2}}{\sum_{n=1}^{f} X_{n}^{2}},
$$

where $X_{n}$ is the APN-AMBR for the $n$ MTC devices. In this description, the bit rate of all traffic generated by a group of MTC devices can be controlled by determining the APNAMBR of each MTC device according to current resource use of the MTC group. Group-based policing regulates the maximum bit rate of all traffic generated, and the total bit rate of traffic generated through all non-GBR bearers connects to the same APN. The MME sends the APN-AMBR to the MTC

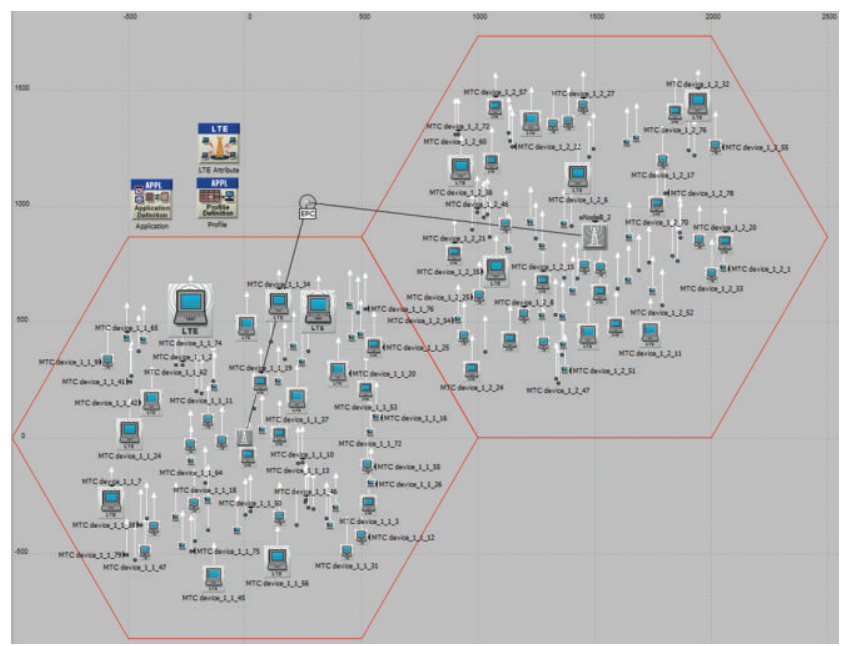

FIGURE 7: Network topology of the simulation.

device requesting attachment by using the PDN connection establishment procedure. The MME may store $G_{i, j}, D_{i}, M_{i}$, $H_{s^{\prime}}$, and $L_{s^{\prime}}$ in individual groups of MTC devices by using a multistage controlled bit rate feature.

\section{Simulation Environment}

The experiments in this study were performed using OPNET Modeler 17.1 with LTE module capability to simulate an MTC environment [20]. The simulation was conducted for 3,600 seconds to investigate the stable state result for all MTC device nodes.

This simulation tests the performance of the proposed mechanism in a typical network consisting of one EPC and 160 randomly distributed MTC device nodes. As Figure 7 shows, this experimental environment is based on a simulated OPNET MTC network topology containing two cells. Each cell has an eNB, and each eNB has 80 MTC 
TABLE 1: LTE simulation system parameters $[17,18]$.

\begin{tabular}{lc}
\hline Parameters & Value \\
\hline PHY profile & FDD \\
Bandwidth & $20 \mathrm{MHz}$ \\
Packet size & 1024 byte \\
Cycle prefix & Normal \\
UL SC-FDMA channel (base frequency) & $1920 \mathrm{MHz}$ \\
DL OFDMA channel (base frequency) & $2110 \mathrm{MHz}$ \\
Max. retransmission (HARQ) & 3 \\
Handover type & Intrafrequency \\
Path-loss parameter & Free space \\
\hline
\end{tabular}

devices. The transmission powers of the MTC device and eNB Node were set to 0.006 watts and 0.012 watts, respectively. The OPNET node models of the eNB and MTC devices are lte_enodeb_atm4_ethernet4_slip4_router and lte_wkstn_adv, respectively. These simulations assume that the moving mode of the MTC devices follows the random waypoint model. The movement speed of each MTC device was uniformly distributed between 1 and $5 \mathrm{~m} / \mathrm{s}$. The fixed eNB node model featured router functionality. The UE node model featured workstation functionality [21]. The global configuration object was used to configure the parameters, such as EPS bearer definitions and PHY profiles, in the LTE attributes node $[17,18]$.

The UEs have a CQI index to provide QoS awareness. The services are grouped into different QoS classes. The main contribution of the proposed mechanism is the MAC layer, whereas the actual physical transmission is adopted from the OPNET LTE model. Table 1 presents a summary of the simulation parameters. This system considers both the downlink and uplink. The MAC layer scheduler implies that the GBR bearers are always allocated radio resources before the non-GBR bearers. The eNB module implements priority scheduling for GBR and non-GBR bearers. Table 2 presents the experimental data for QoS class services. The MTC traffic on the EPS bearer is generated between the eNB and MTC devices [19]. The EPS bearer configuration attribute defines four bearers: platinum, gold, silver, and bronze.

\section{Results and Discussion}

Figure 8 shows a comparison of the blocking probability and traffic load of the MTC application, validating the analytical results by simulation. The general distribution is set according to the assumption that $20 \%$ of grant time intervals are long (with a service rate of 1.2), 30\% of grant time intervals are short (with a service rate of 0.9 ), and the remaining $40 \%$ grant time intervals have a service rate of 1 . Simulation results indicate that saturation occurs when the number of MTC devices reaches 80 and $\rho>0.002$ (using the MTC application in the LTE system). Saturation means that all grant time intervals of MTC devices have been blocked. However, the lower-priority grant time intervals cannot be scheduled when the number of MTC devices increases, and the blocking probability is more than 1 . To prevent the transmission of

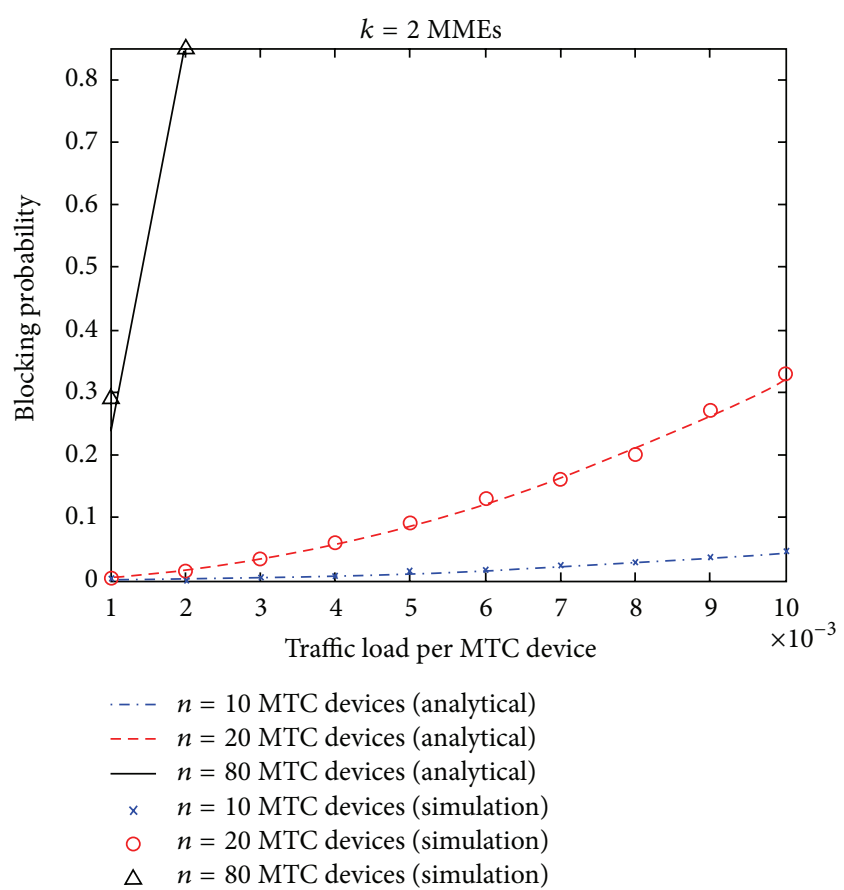

FIGURE 8: Comparisons of blocking probability, and traffic load.

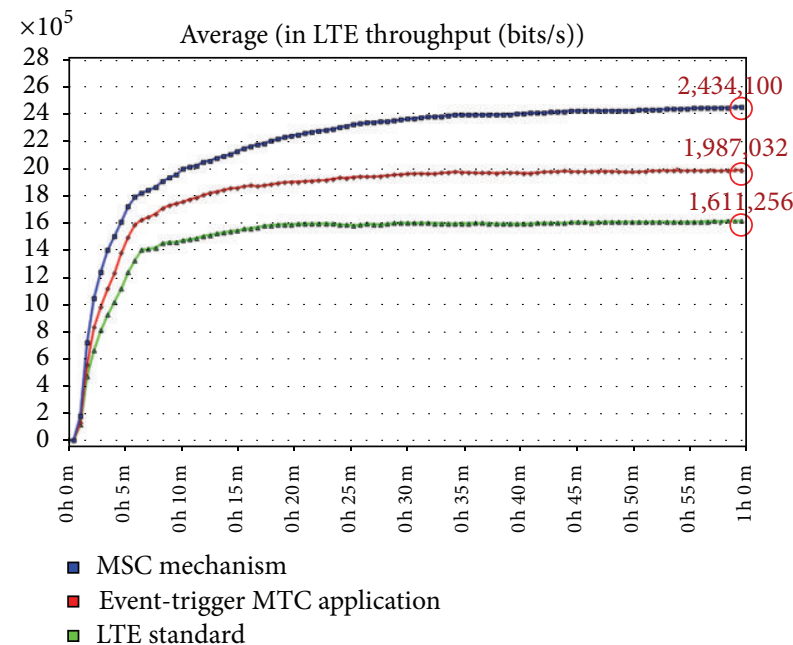

FIGURE 9: System throughput in the simulation (in bits/second).

blocked MTC devices from wasting AMBR, it is necessary to periodically verify the inaccessible bandwidth from the MME.

Figure 9 shows the system throughput for the LTE standard, Event-Trigger MTC application, and our proposed MSC mechanism. The simulation results in Figure 9 indicate that saturation occurs when the simulation time reaches 1,800 seconds. Saturation indicates that all the MTC devices of one eNB have been scheduled. The Event-Trigger MTC application reserves the extra bandwidth and reallocates the remaining bandwidth. Using the Event-Trigger scheme, the maximal average throughput reaches $2 \mathrm{Mbps}$ before the system becomes saturated. The MSC mechanism with a MTC 
TABLE 2: Standardized QCI characteristics [19].

\begin{tabular}{|c|c|c|c|c|c|}
\hline Service class & Priority & Type & Packet delay budget & Packet error loss rate & Guaranteed bit rate \\
\hline \multirow{2}{*}{ Platinum } & 1 & Non-GBR & $100 \mathrm{~ms}$ & $10^{-6}$ & - \\
\hline & 2 & GBR & $100 \mathrm{~ms}$ & $10^{-2}$ & $128 \mathrm{kbps}$ \\
\hline \multirow{2}{*}{ Gold } & 3 & GBR & $50 \mathrm{~ms}$ & $10^{-3}$ & $858 \mathrm{kbps}$ \\
\hline & 4 & GBR & $150 \mathrm{~ms}$ & $10^{-3}$ & $384 \mathrm{kbps}$ \\
\hline \multirow{2}{*}{ Silver } & 5 & GBR & $300 \mathrm{~ms}$ & $10^{-6}$ & $384 \mathrm{kbps}$ \\
\hline & 6 & Non-GBR & $300 \mathrm{~ms}$ & $10^{-6}$ & - \\
\hline \multirow{2}{*}{ Bronze } & 7 & Non-GBR & $100 \mathrm{~ms}$ & $10^{-3}$ & - \\
\hline & 8 & Non-GBR & $300 \mathrm{~ms}$ & $10^{-6}$ & - \\
\hline
\end{tabular}

application and a multistage controlled process achieves higher performance because it has a higher average throughput. This is the main reason for obtaining the time period report information and an appropriate bandwidth-controlled feature. After the bandwidth allocation reaches an improved state because of the resource use, the MTC device can transmit at a higher data rate using previously inaccessible bandwidth. Because the MSC mechanism considers the method of allocation of the APN-AMBR, the MTC device can select the appropriate APN-AMBR with a superior MTC application to the data transmission, thereby achieving a maximal average throughput of $2.43 \mathrm{Mbps}$. This phenomenon demonstrates that the proposed MSC mechanism markedly improves throughput compared to the LTE standard because of the extra bandwidth consumed and the inferior groupbased control method of the LTE standard. The final values of throughput after 3,600 seconds in the simulation are 1,611,256 bps for LTE standard system, 1,987,032 bps for Event-Trigger MTC application, and 2,434,100 bps for MSC mechanism. Compared to the LTE system with Event-Trigger MTC application, the MSC mechanism achieves a $22.5 \%$ higher throughput in this simulation.

Figure 10 shows the relationships among the packet delay times of the MTC application. For the Event-Trigger MTC application, the MME requests an additional period without modifying resource use at the HSS. Additional periods cause the time period report time to increase as the delay time increases. The final values of packet delay time after 3,600 seconds in the simulation are 0.123 seconds for LTE standard system, 0.132 seconds for Event-Trigger MTC application, and 0.100 seconds for MSC mechanism. Compared to the LTE standard and Event-Trigger MTC application, the packet delay time of the MSC mechanism achieves higher performance in the LTE system because each MME continuously monitors its APN-AMBR. The HSS gathers the APN-AMBR information received through the create session request messages sent by the MMEs. When the groupbased policing is optimal or the resource use is low, the MTC application should also be served using the MSC mechanism.

Figure 11 shows the simulation results of Jain's fairness index value at various MTC traffic applications for 80 nodes, indicating that all of the MTC traffic applications in this paper have relatively high Jain's fairness index values $(0.62$ $<$ FI). This chart shows that the MSC mechanism has a higher fairness than the LTE standard and Event-Trigger

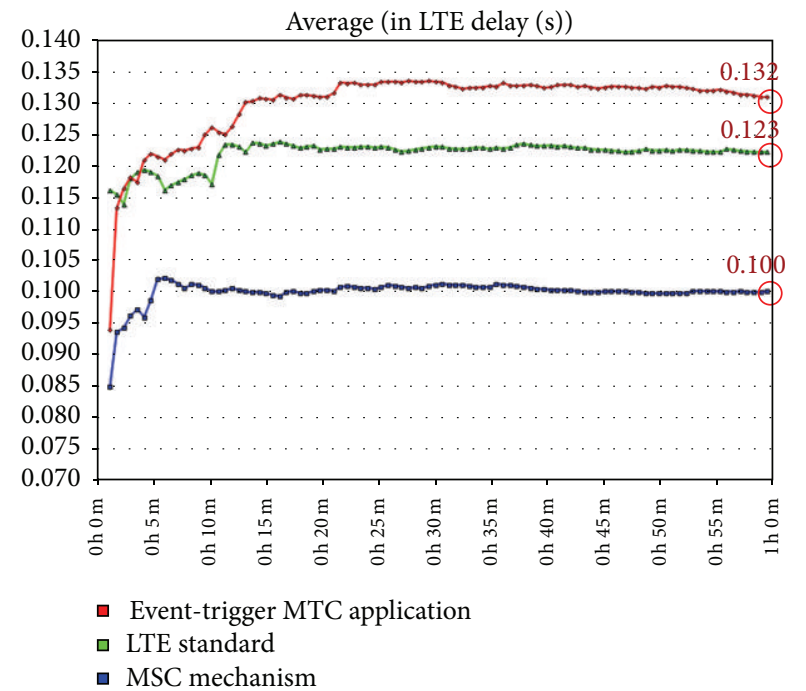

FIGURE 10: Packet delay time of the MTC application in the simulation (in seconds).

MTC application. For the MSC mechanism, the fairness of MTC traffic applications depends on the amount of traffic transmitted by the selected APN-AMBR. This is the main reason the MSC mechanism has more resource utilization. For the transmission model of an Event-Trigger MTC application, a number of MTC traffic applications are reallocated a portion of bandwidth by the attach reject message. In the MTC traffic type, the fairness index of Event-Trigger MTC application is higher than that of the LTE standard because the Event-Trigger MTC application considers the method of allocating AMBR based on the modified period and the amount of controlled time.

Markov chain with $M / G / k / k$ and Jain's fairness index are used to analyze machine-type communication in a 3GPP network. Table 3 compares the results between analysis and simulation of Jain's fairness index (FI) values, which shows that analysis can be validated by simulation.

\section{Conclusion}

This study proposes an MSC mechanism in an LTE system to process the operations of bandwidth allocation based 
TABLE 3: Comparison of results between analysis and simulation of Jain's fairness index (FI) value.

\begin{tabular}{lcccccrrr}
\hline Service class & \multicolumn{2}{c}{ Gold } & \multicolumn{2}{c}{ Silver } & \multicolumn{2}{c}{ Bronze } & \multicolumn{2}{c}{ Platinum } \\
Simulation or analysis & Sim. & Ana. & Sim. & Ana. & Sim. & Ana. & Sim. & Ana. \\
\hline MSC mechanism & 0.89 & 0.89 & 0.80 & 0.79 & 0.72 & 0.70 & 0.71 \\
Event-Trigger MTC application & 0.89 & 0.89 & 0.79 & 0.79 & 0.70 & 0.70 & 0.70 \\
LTE standard & 0.89 & 0.89 & 0.76 & 0.75 & 0.67 & 0.66 & 0.63 & 0.63 \\
\hline
\end{tabular}

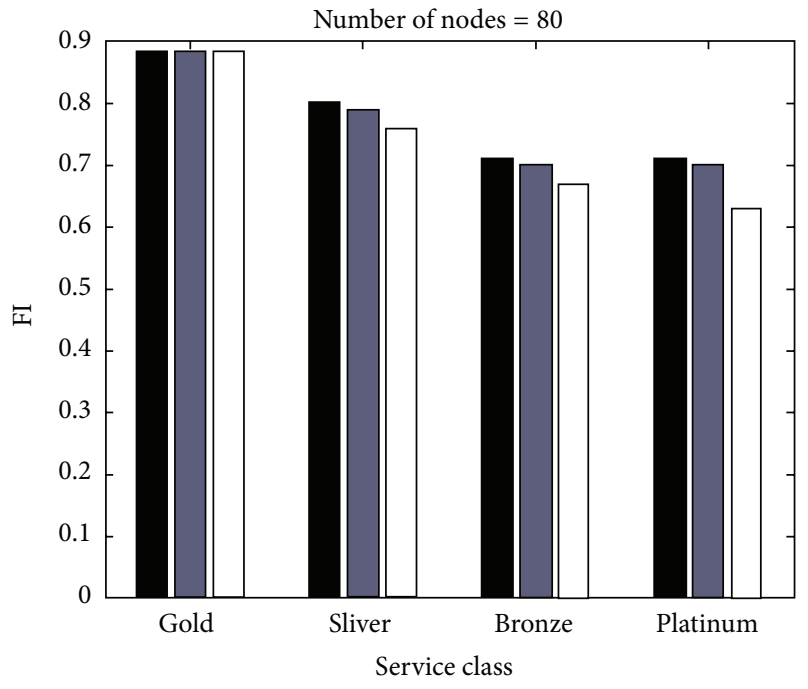

MSC mechanism
Event-Trigger MTC application
LTE standard

FIGURE 11: The comparison of Jain's fairness index (FI) values.

on MTC application, QoS parameters, and the bandwidth requirements of MTC devices and the eNB. This study presents a comparison of the performance of the proposed MSC mechanism and the LTE standard mechanism of MTC application. The service flow simulations of the OPNET modeler indicate the MSC mechanism achieves a higher system throughput, a lower delay time, and greater longterm fairness for multiple MTC devices. Experimental results indicate that the throughput of the MSC mechanism is $22.5 \%$ higher than that of the LTE standard model using the group-based policing, which implies that the proposed MSC mechanism is an effective bandwidth allocation method in an LTE system with MTC devices.

\section{Conflict of Interests}

No conflict of interests exists between the authors and all of the mentioned organizations or corporations, including 3GPP and OPNET Modeler. The 3rd Generation Partnership Project (3GPP) is a nonprofit international telecommunication standard development organization, which defines new-generation telecommunication standards including LTE (long-term evolution). In this study, we perform the simulation by OPNET Modeler ver. 17.1, which is a popular simulation tool for analyzing and designing communication networks, devices, protocols, and applications. They have paid the licensing fee for legal use of OPNET Modeler ver. 17.1 in our research.

\section{Acknowledgment}

A special thanks goes to Institute for Information Industry in Taiwan, for its great help in supporting the simulation environment and key LTE simulation modules to this study.

\section{References}

[1] P. Jain, P. Hedman, and H. Zisimopoulos, "Machine type communications in 3GPP systems," IEEE Communications Magazine, vol. 50, no. 11, pp. 28-35, 2012.

[2] T. Taleb and A. Kunz, "Machine type communications in 3GPP networks: potential, challenges, and solutions," IEEE Communications Magazine, vol. 50, no. 3, pp. 178-184, 2012.

[3] O. Del Rio Herrero and R. De Gaudenzi, "High efficiency satellite multiple access scheme for machine-to-machine communications," IEEE Transactions on Aerospace and Electronic Systems, vol. 48, no. 4, pp. 2961-2989, 2012.

[4] K. Chang, A. Soong, M. Tseng, and Z. Xiang, "Global wireless machine-to-machine standardization," IEEE Internet Computing, vol. 15, no. 2, pp. 64-69, 2011.

[5] 3GPP, "Service Requirements for Machine-Type Communications, TS 22.368 V12.0.0," 2012.

[6] A. Ksentini, Y. Hadjadj-Aoul, and T. Taleb, "Cellular-based machine-to-machine: overload control," IEEE Network, vol. 26, no. 6, pp. 54-60, 2012.

[7] 3GPP, "System Improvements for Machine-Type Communications, TR 23.888 V11.0.0," 2012.

[8] K. Zheng, F. Hu, W. Wang, W. Xiang, and M. Dohler, "Radio resource allocation in LTE-advanced cellular networks with M2M communications," IEEE Communications Magazine, vol. 50, no. 7, pp. 184-192, 2012.

[9] C. Songyean, R. Heetae, L. Jangwon, B. Beomsik, L. Chaegwon, and J. Sangsoo, "Group-based control method and apparatus for MTC devices in mobile communication system," United States patent US, 2012/0209978, 2012.

[10] S. Lien and K. Chen, "Massive access management for QoS guarantees in 3GPP machine-to-machine communications," IEEE Communications Letters, vol. 15, no. 3, pp. 311-313, 2011.

[11] Y. Chen and W. Wang, "Machine-to-machine communication in LTE-A," in Proceedings of IEEE 72nd Vehicular Technology Conference Fall (VTC '10-Fall), pp. 1-4, Ottawa, Canada, September 2010.

[12] S. Y. Lien, K. C. Chen, and Y. Lin, "Toward ubiquitous massive accesses in 3GPP machine-to-machine communications," IEEE Communications Magazine, vol. 49, no. 4, pp. 66-74, 2011. 
[13] 3GPP, "GPRS enhancements for E-UTRAN access, TS 23.401 V11.4.0," 2012.

[14] G. Wu, S. Talwar, K. Johnsson, N. Himayat, and K. D. Johnson, "M2M: from mobile to embedded internet," IEEE Communications Magazine, vol. 49, no. 4, pp. 36-43, 2011.

[15] D. Gross and C. M. Harris, Fundamentals of Queuing Theory, Wiley, New York, NY, USA, 3rd edition, 1998.

[16] R. Jain, D. M. Chiu, and W. Hawe, "A quantitative measure of fairness and discrimination for resource allocation in shared system," DEC Research Report TR-301, 1984.

[17] M. Torad, A. El Qassas, and H. Al Henawi, "Comparison between LTE and WiMAX based on system level simulation using OPNET modeler (release 16)," in Proceedings of 28th National Radio Science Conference (NRSC '11), pp. 1-9, Cairo, Egypt, April 2011.

[18] K. Andersson, S. A. M. Mostafa, and R. Ui-Islam, "Mobile VoIP user experience in LTE," in Proceedings of IEEE 36th Conference on Local Computer Networks (LCN '11), pp. 785-788, Bonn, Germany, October 2011.

[19] 3GPP, "Policy and charging control architecture, TS 23.203 V11.8.0," 2012.

[20] “OPNET LTE Specialized Model," http://www.opnet.com/LTE/.

[21] B. H. Lee and S. L. Kim, "Mobility control for machine-tomachine LTE systems," in Proceedings of Wireless Conference 2011-Sustainable Wireless Technologies (European Wireless), pp. 1-5, Vienna, Austria, April 2011. 


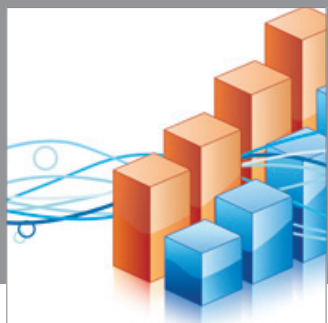

Advances in

Operations Research

mansans

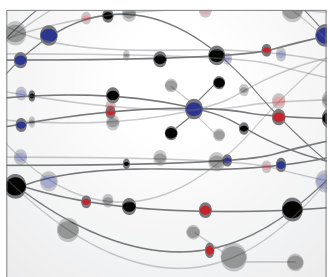

The Scientific World Journal
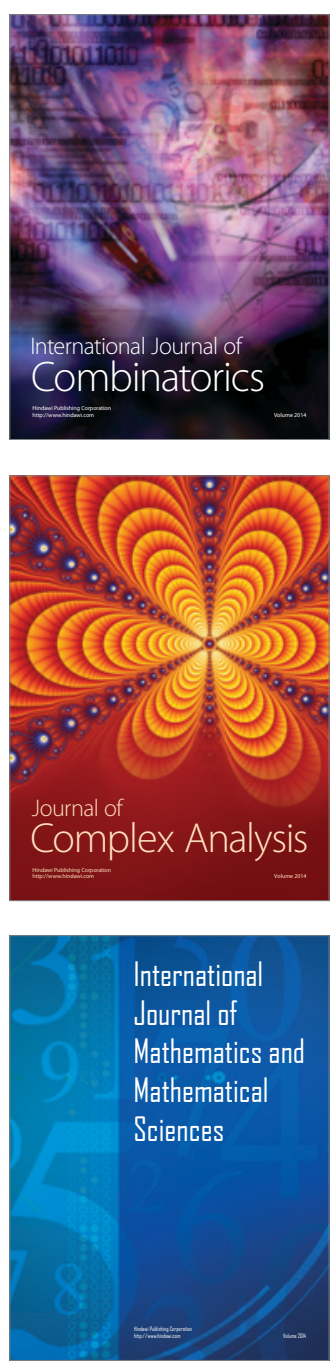
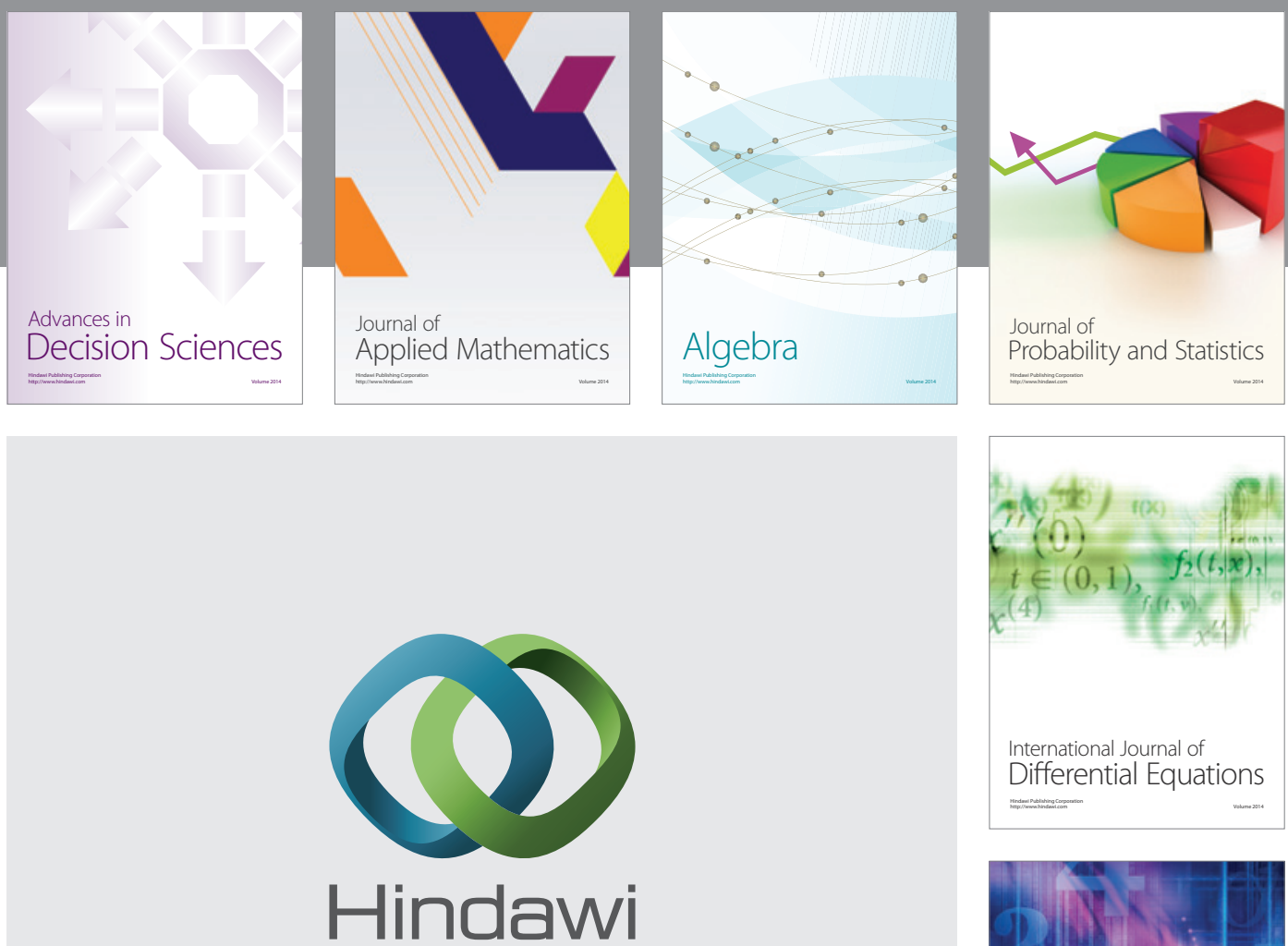

Submit your manuscripts at http://www.hindawi.com
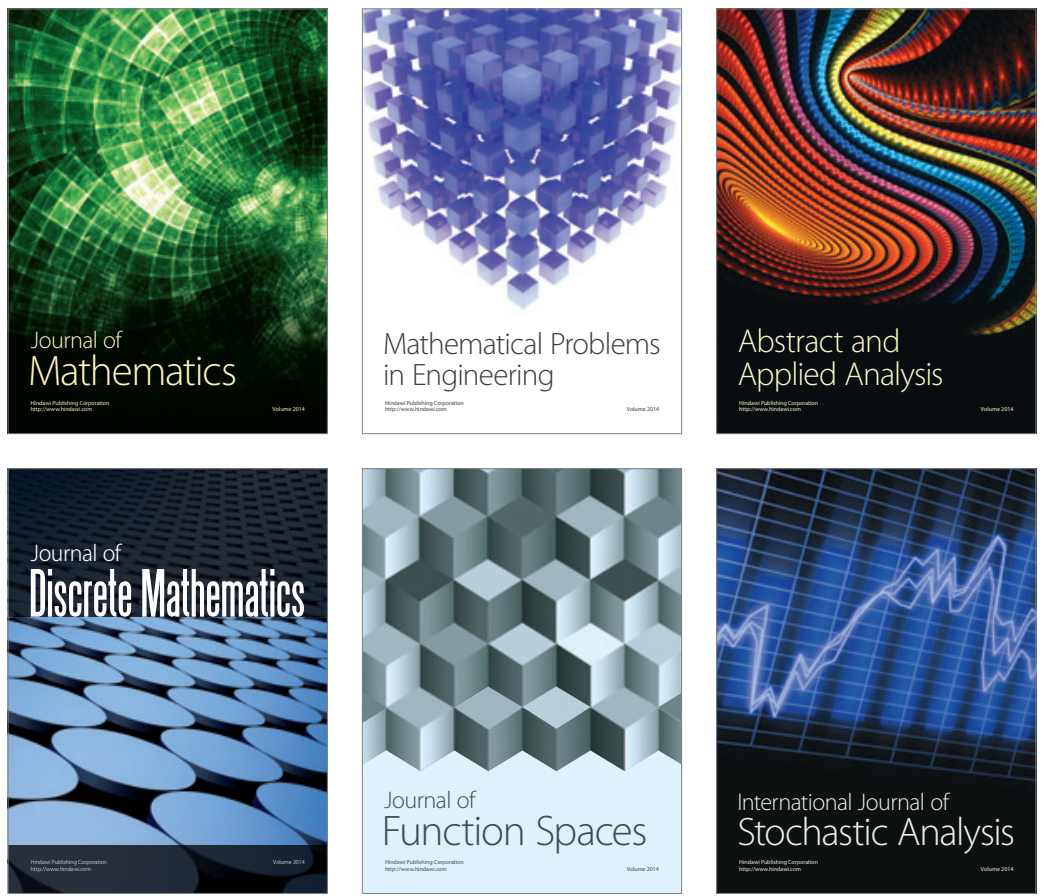

Journal of

Function Spaces

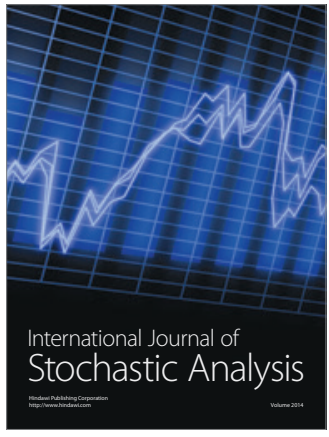

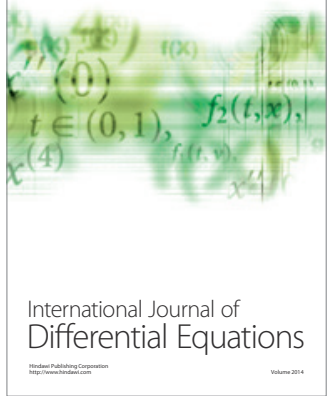
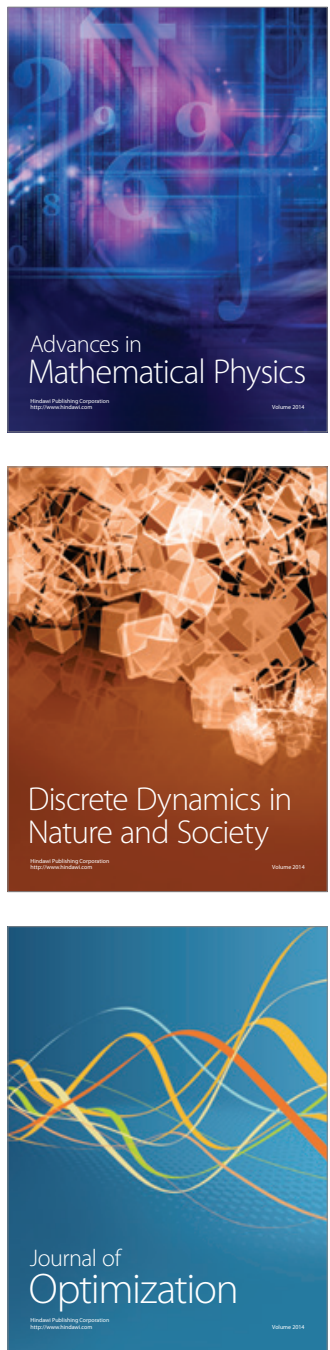